on how to administer nutrition at home. Children monitored have progressively grown in height and weight.

690

\section{IMPLEMENTING "NURSE-TALKS" WITH \\ PARENTS IN ORDER TO ENSURE AND QUALIFY COMMUNICATION WITH A SPECIFIC INTEREST IN THE PARENTAL PERSPECTIVE}

\author{
H. Haslund \\ NICU, Aalborg Hospital, Aarhus University \\ Hospital, Aalborg, Denmark
}

Background: In NICU communication with parents is a central part of nurse practice.

Method: In NICU Aalborg we tested if weekly conversations between parents and the nurse would better the parents experience of coherence and meaning during their long stay. The main focus of these conversations was to actively explore the parental perspective. The information conversation (often between doctor and patient) was something quite different.

We wanted to facilitate talking and elaborated a paper with suggestions of themes and subjects. This made the nature of the conversation clear to nurse and parents. During the conversation new aggrements were made for the comming week, in order to prevent the parental stess that often is present partly due to unclear role-reponsabilty sharing between parents and nurses (there is a major shift during a stay in Nicu with parents gaining confidence, knowledge and responsability as time goes by)

We conducted a survey (quesstionnaire) among nurses and parents in order to gather information from nurses and parents about the benefit of communiction within this specific frame.

Results: The results showed that parents as well as nurses found this form of communiction highly usefull. Parents found it most helpfull and nurses gathered valuable information from the parents. Using the inspirationpaper was thus evauated as a very helpfull tool and the form of communiction also very beneficial.

\section{BECOMING A NIDCAP TRAINER - IMPLEMENTATION OF INFANT-ORIENTED AND FAMILY-CENTERED CARE IN A NICU}

\section{E. Jørgensen}

\section{Neonatology A6, Aarhus University Hospital, Skejby, Aarhus, Denmark}

A premature born infant is active in coping with her and his own development after a premature birth. The infant has to adjust to the new environment and new experiences. The infant is depending on parents, health care professionals and the surrounding environment to help it regulate the challenges a premature birth gives.

The NIDCAP $®$ (Newborn Individualized Developmental Care and Assessment Program) is a family-centered and individualized program tailored to infant's levels of stability to reach optimal health and development.

The aim is to:

- Bring infant-oriented and family-centered care into the NICU, challenging health care professionals and the parents to support the infant's own efforts to reach optimal health and development.

- Provide the healthcare professionals with professional NIDCAP skills

- In the future become a NIDCAP Training center

Methods: Training in the NIDCAP approach, to ensure full multi-disciplinary implementation.

The Training consists of lectures, workdays with NIDCAP Master Trainers, independent self preparation and practice and a reliability test and certification.

\section{Expectations:}

- The goal of education and training in the developmental approach to care is to bring about a shift from protocol-based and task-oriented care to strategic process thinking and relationship-based care.

- Is to allow the infant to focus on her or his energy on sleeping and growing.

- Support the parents as the most important caregivers for their child. 